\title{
Assess Reliability Parameters of an Electronic Voting Machine using a Neural Network Technique
}

\author{
C. M. Batra, Ritu Gupta, Ekata
}

\begin{abstract}
The structure of Electronic Voting Machine (EVM) is an interconnected network of discrete components that record and count the votes of voters. The EVM system consists of four main subsystems which are Mother board of computer, Voting keys, Database storage system, power supply (AC and DC) along with various conditions of functioning as well as deficiency. The deficiency or failure of system is due to its components (hardware), software and human mismanagement. It is essential to reduce complexity of interconnected components and increase system reliability. Reliability analysis helps to identify technical situations that may affect the system and to predict the life of the system in future. The aim of this research paper is to analyze the reliability parameters of an EVM system using one of the approaches of computational intelligence, the neural network $(N N)$. The probabilistic equations of system states and other reliability parameters are established for the proposed EVM model using neural network approach. It is useful for predicting various reliability parameters and improves the accuracy and consistency of parameters. To guarantee the reliability of the system, Back Propagation Neural Network (BPNN) architecture is used to learn a mechanism that can update the weights which produce optimal parameters values. Numerical examples are considered to authenticate the results of reliability, unreliability and profit function. To minimize the error and optimize the output in the form of reliability using gradient descent method, authors iterate repeatedly till the precision of 0.0001 error using MATLAB code. These parameters are of immense help in real time applications of Electronic Voting Machine during elections.
\end{abstract}

Keywords: Back Propagation algorithm, EVM, Gradient descent method, Human failure, Neural Network approach, Neural weights, Profit function, Reliability, unreliability.

\section{INTRODUCTION}

To guarantee the privilege of the right to vote of the citizens, the electoral procedure with security and integrity is a fundamental condition of any nation. According to the People's Representation Act of 1951 and under article 19 (1) (a) of the Constitution of India, voting is a constitutional and fundamental right. Elections in India are conducted almost

Revised Manuscript Received on July 10, 2020.

*Correspondence Author

C. M. Batra, Department of Applied Sciences, KIET Group of Institutions, Ghaziabad, India. E-mail: cm.batra@kiet.edu

Ritu Gupta, Department of Applied Sciences, KIET Group of Institutions, Ghaziabad, India. E-mail: ritu.gupta@kiet.edu

Ekata*, Department of Applied Sciences, KIET Group of Institutions, Ghaziabad, India. E-mail: ekata@kiet.edu

(C) The Authors. Published by Blue Eyes Intelligence Engineering and Sciences Publication (BEIESP). This is an open access article under the CC BY-NC-ND license (http://creativecommons.org/licenses/by-nc-nd/4.0/) entirely using electronic voting machines (EVMs) built over the past two decades. As India is a democratic nation, these devices have been praised for its easy designing, handling and reliability [1]-[2]. But several specifications of machineries design and complexity of system do not disclose, that may cause degradation in functioning or failure of the system [4]. Some other reasons of failure of system are human mishandling. To solve the reliability problems, it is necessary to identify hypotheses/ constraints of dependencies or interdependencies of units/ components of the system [3][6] To increase the system reliability, standby unit(s) with/ without waiting time to repair is appended [8]. We have already studied and analyze reliability parameters of these type of systems in past, using the technique of supplementary variables. Numerous studies on reliability and profit of systems have been carried out to analyze the behavior of the system, with respect to time. Several reliability parameters of a multi-component complex system under common cause failure, failure due to human error etc. have been studied by renowned researchers [16]. There are many methodologies/ techniques used to evaluate the system reliability. Some of these are Petri nets, supplementary variable technique, stochastic reward nets, and regenerative point technique etc. [5], [7]-[9]. All of these conventional methods to evaluate the reliability parameters precisely, but the current demand is to update the output at the extend of optimization and minimization of errors.

Neural network (NN) learning algorithm has been designed in 1943 by a neurophysiologist, Warren McCulloch and a young mathematician, Walter Pitts [15]. The basic structure of NN relies on the biological structure of neurons in human brain to recognize the input patterns associated with their output. As well as, neural network is dynamic and predict new outcomes from past experience at high speed and minimize the errors. In view of this, supervised learning of neural network approach can be utilized to solve the mathematical model of multi-component system and optimize the results as required by system designers. A neural network involves simple processing elements, neurons that are well connected by weighted synaptic links and form network architecture (Figure 1). Authors proposed architecture of neural network comprises three layers, which incorporate the following: Input layer that takes input in context of problem;

Hidden layer particular to update the weights in between input and output layers and

Output layer manifests the optimized results to the supervisor. It presents a complex global behavior,

\section{Published By:}

Blue Eyes Intelligence Engineering and Sciences Publication

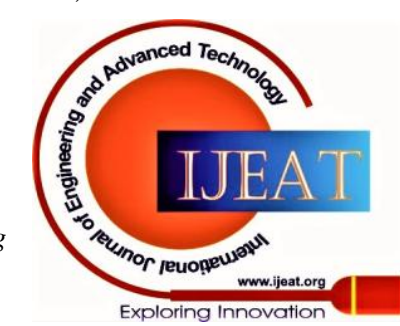


determined by the connections between the input elements, processing elements and output parameters along the learning mechanism to train the system. The learning algorithms characterize the process of adjusting/ updating the weights by adopting activation function and minimizing the errors of the weights using error correction gradient descent method in back propagation algorithm. The back propagation algorithm in neural network (BPNN) was introduced in 1970s and used for training the system network and minimizing the error up to required accuracy. Now, Neural Networks (NN) has been applied in evaluation of reliability parameters as well as simulates its growth [10]-[12]. In this paper, we propose an NN based reliability growth model based on EVM system. The results shown in numerical example includes initial values and optimized values of the reliability, unreliability, and profit function. The proposed BPNN approach is a promising tool for stochastic analysis of system reliability by minimizing the error up to desired negligible accuracy and optimizes the results.

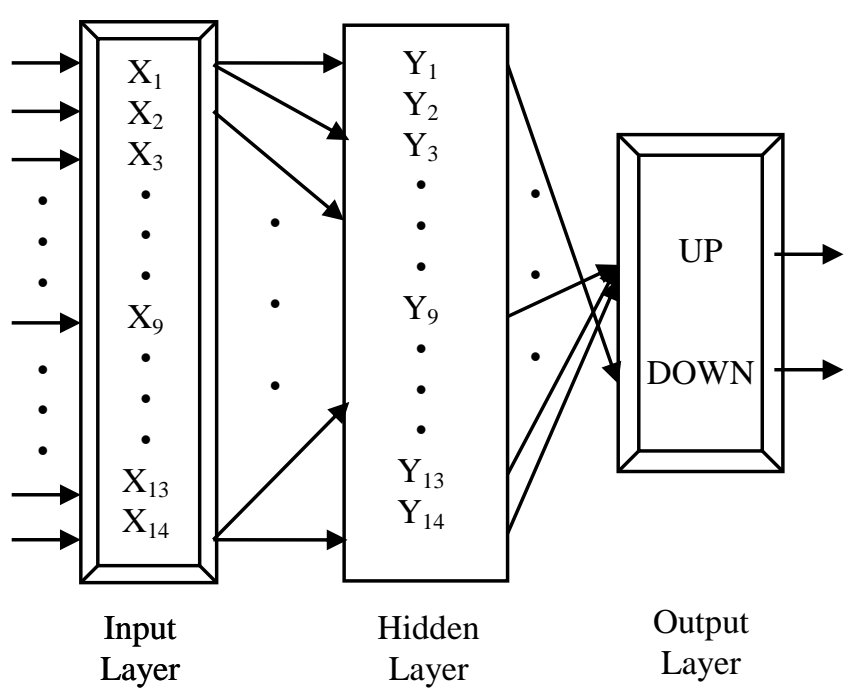

Fig. 1: Neural Networks Structure

\section{SYSTEM DESCRIPTION}

From many decades, countries are governed by their citizens. People have authority to choose their governing bodies those serve the country as well as save their rights i.e. Democracy. An election is a procedure by which representative of people elected. Elections are a defining feature of democratic government since $17^{\text {th }}$ century [1]-[2]. Voting is, the only, method for people to elect their representative by collective decision and to express an opinion. In earlier time, name of all candidates, who wanted to serve the country, written on a paper with their specific symbol i.e. ballot paper. Voters, people who have right to vote; cast the ballot paper for their chosen candidates. Electorate can directly make decisions after collecting and counting all casted ballots and declare the constituency. This manual process was time taking and sometimes became the reason of riot. In view of all these problems, Electronic voting machine is introduced that uses electronic means to take care of casting and counting of votes. Input of votes, recording it, data encryption, counting of votes and transmission to main server is a rapid process. E-voting machine may be used standalone by the connection with computers and internet. It can convert e-report to tabulated results easily in less time and with more accuracy [13]-[14].

Electronic Voting Machine consists of

1. Subsystem 1 (A)- Mother Board: It consists of Micro controller (Mc), Integrated Circuits, latch and decoders(D), buzzer(B), LCD display, LED green(LED), RAM reset (Re) and RAM check (Rc).

2. Subsystem 2 (V)- Voting Kit: It connects with subsystem A through wires (W)

3. Subsystem 3 (DSS)- Database Storage System: It consists of two units viz. personal computer (PC) and scanner(SCAN) and connected to subsystem A through interface (a parallel port)

4. Subsystem 4 (PS)- Power Supply: There are two type of power supplies i.e. through alternating current (PSAC) and DC (PSDC)

The unit Mc is connected to B, LCD and LED through wires. The integrated circuit is connected to Re and Rc. It is assumed that subsystems Mc, IC's and D are non volatile, i.e. they are in operable state whenever the system is required. Subsystem $\mathrm{V}$ is connected to unit Mc, B, LCD and LED through wires. Subsystem DSS consists of two units PC \& Scanner that is connected to unit IC's, Re and Rc through interface (a parallel port). Wires connect all the subsystems and their units. Fixed power supply is given to the system (Figure 2). Failure of B and LCD reduces the efficiency of the system, causing a degraded state while failure of both units, results into a non operable state of the system. The whole complex system can also be in a total failure state due to failure of unit LED / Rc/ Re or any key in subsystem B / PC/ SCAN and due to human error.

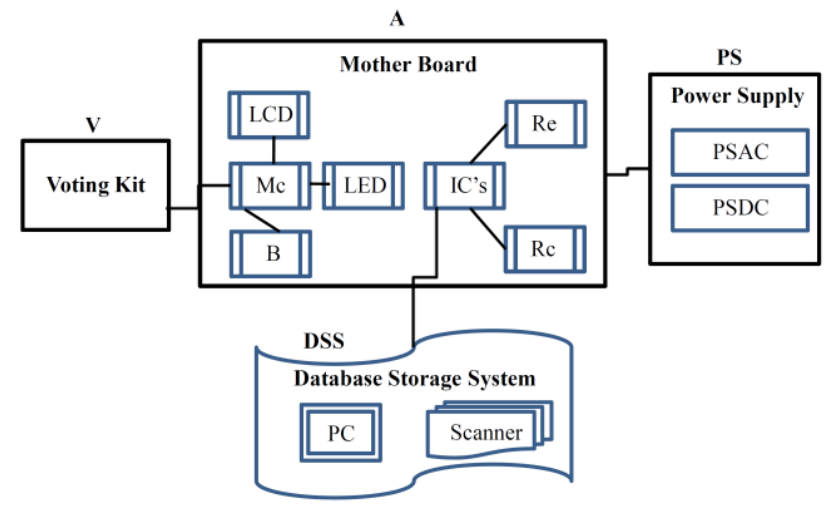

Fig 2 : Block Diagram

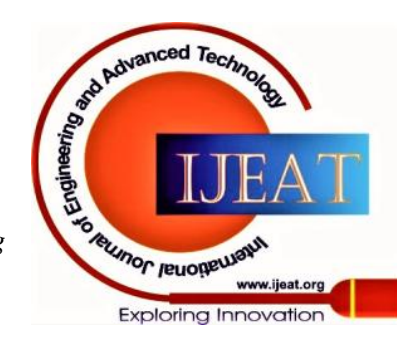




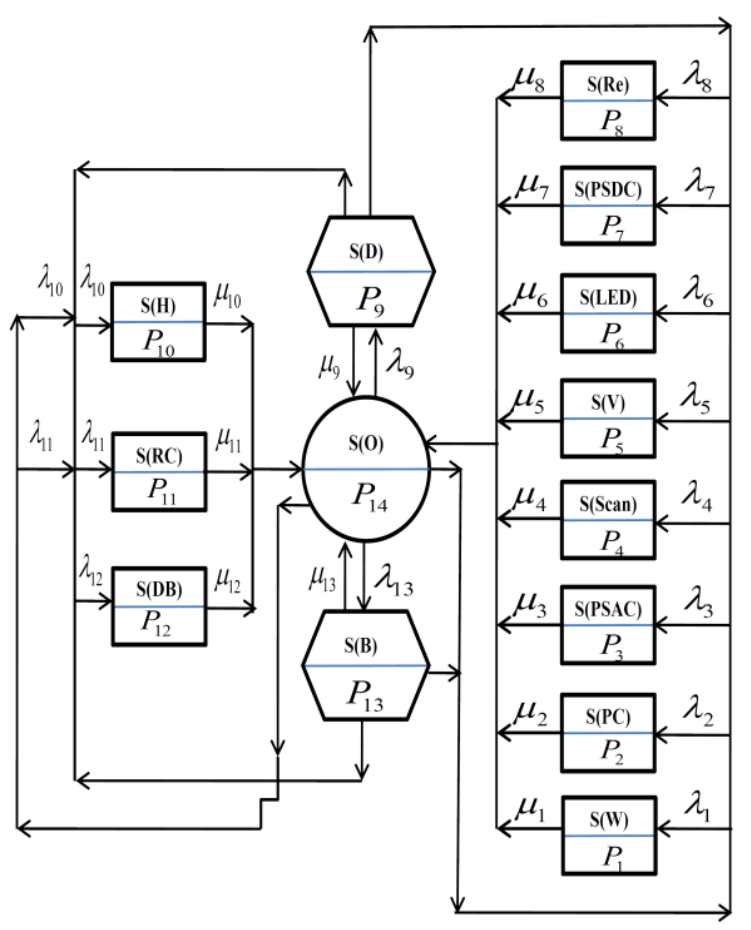

Fig. 3: Transition State Diagram

Authors established the probabilistic equation of mathematical model for electronic voting machine system from transition state diagram and evaluate reliability parameters using neural network technique. All hardware/ human failures and repairs are exponentially distributed and treated as synaptic weights in NN. Rajasekaran et. al. [10] and many others described the back propagation algorithm in neural networks to improve the results. The learning mechanism in Back propagation of neural network is used to update the weights in several iterations as these parameters produce better results. .m file code in MATLAB has been developed to predict the reliability, unreliability and profit function using Back propagation neural network technique.

\section{ASSUMPTIONS}

1. Initially, all subsystems of system are good.

2. During the degraded state of the system, failure of unit B/ LCD may cause the failure of other units.

3. The system fails completely due to failure of LED, Re, Rc, subunits PC and scanner and failure of units B and LCD simultaneously.

4. Since the units are connected through wire, so any disconnection or any fuse wire may result in breakdown of the machine (system).

5. The system may also fail if there is a power cut of DC or AC supply of the machine.

6. System fails completely due to mishandling i.e. a human error

7. All failures and repairs of system are exponentially distributed.

8. Failures and repairs of the system are established as synaptic weights.

9. The repaired subsystem(s) function as new.

\section{NOTATIONS}

$\begin{array}{ll}P_{i}(t) & \begin{array}{l}i^{\text {th }} \text { state Probability at any time } t, \\ \end{array} \\ P_{i}(t+\Delta t) & \begin{array}{l}i^{t h} \text { state Probability at time }(t+\Delta t), \\ \end{array} \\ \lambda_{i} & \begin{array}{l}\text { Hardware failure, due to } i \text { th } 2,3 \ldots, 14 \\ \text { failure, } i=1,2, \ldots, 13\end{array} \\ \mu_{i} & i \text { th unit Hardware repair, } \\ & i=1,2, \ldots, 13\end{array}$

\section{SOLUTION OF THE MODEL}

The reliability and profit function of the system have been explored by neural network approach. The various states of system can be described using probabilistic phenomenon. These system state probabilities are as follows:

$$
\begin{aligned}
& P_{1}(t+\Delta t)=\left(1-\mu_{1} \Delta t\right) P_{1}(t)+\lambda_{1} \Delta t P_{9}(t) \\
& +\lambda_{1} \Delta t P_{13}(t)+\lambda_{1} \Delta t P_{14}(t) \\
& P_{2}(t+\Delta t)=\left(1-\mu_{2} \Delta t\right) P_{2}(t)+\lambda_{2} \Delta t P_{9}(t) \\
& +\lambda_{2} \Delta t P_{13}(t)+\lambda_{2} \Delta t P_{14}(t) \\
& P_{3}(t+\Delta t)=\left(1-\mu_{3} \Delta t\right) P_{3}(t)+\lambda_{3} \Delta t P_{9}(t) \\
& +\lambda_{3} \Delta t P_{13}(t)+\lambda_{3} \Delta t P_{14}(t) \\
& P_{4}(t+\Delta t)=\left(1-\mu_{4} \Delta t\right) P_{4}(t)+\lambda_{4} \Delta t P_{9}(t) \\
& +\lambda_{4} \Delta t P_{13}(t)+\lambda_{4} \Delta t P_{14}(t) \\
& P_{5}(t+\Delta t)=\left(1-\mu_{5} \Delta t\right) P_{5}(t)+\lambda_{5} \Delta t P_{9}(t) \\
& +\lambda_{5} \Delta t P_{13}(t)+\lambda_{5} \Delta t P_{14}(t) \\
& P_{6}(t+\Delta t)=\left(1-\mu_{6} \Delta t\right) P_{6}(t)+\lambda_{6} \Delta t P_{9}(t) \\
& +\lambda_{6} \Delta t P_{13}(t)+\lambda_{6} \Delta t P_{14}(t) \\
& P_{7}(t+\Delta t)=\left(1-\mu_{7} \Delta t\right) P_{7}(t)+\lambda_{7} \Delta t P_{9}(t) \\
& +\lambda_{7} \Delta t P_{13}(t)+\lambda_{7} \Delta t P_{14}(t) \\
& P_{8}(t+\Delta t)=\left(1-\mu_{8} \Delta t\right) P_{8}(t)+\lambda_{8} \Delta t P_{9}(t) \\
& +\lambda_{8} \Delta t P_{13}(t)+\lambda_{8} \Delta t P_{14}(t) \\
& P_{9}(t+\Delta t)=\left(1-\left(\begin{array}{l}
\lambda_{1}+\lambda_{2}+\lambda_{3}+\lambda_{4}+\lambda_{5} \\
+\lambda_{6}+\lambda_{7}+\lambda_{8}+\lambda_{10} \\
+\lambda_{11}+\lambda_{12}+\mu_{9}
\end{array}\right) \Delta t\right) P_{9}(t) \\
& +\lambda_{9} \Delta t P_{14}(t) \\
& P_{10}(t+\Delta t)=\left(1-\mu_{10} \Delta t\right) P_{10}(t)+\lambda_{10} \Delta t P_{9}(t) \\
& +\lambda_{10} \Delta t P_{13}(t)+\lambda_{10} \Delta t P_{14}(t) \\
& P_{11}(t+\Delta t)=\left(1-\mu_{11} \Delta t\right) P_{11}(t)+\lambda_{11} \Delta t P_{9}(t) \\
& +\lambda_{11} \Delta t P_{13}(t)+\lambda_{11} \Delta t P_{14}(t) \\
& P_{12}(t+\Delta t)=\left(1-\mu_{12} \Delta t\right) P_{12}(t)+\lambda_{12} \Delta t P_{9}(t) \\
& +\lambda_{12} \Delta t P_{13}(t)
\end{aligned}
$$

Published By:

Blue Eyes Intelligence Engineering and Sciences Publication

(c) Copyright: All rights reserved. 


$$
\begin{aligned}
P_{13}(t+\Delta t) & =\left(1-\left(\begin{array}{l}
\lambda_{1}+\lambda_{2}+\lambda_{3}+\lambda_{4}+\lambda_{5} \\
+\lambda_{6}+\lambda_{7}+\lambda_{8}+\lambda_{10} \\
+\lambda_{11}+\lambda_{12}+\mu_{13}
\end{array}\right) \Delta t\right) P_{9}(t) \\
& +\lambda_{13} \Delta t P_{14}(t) \\
P_{14}(t+\Delta t)= & \left(1-\left(\begin{array}{l}
\lambda_{1}+\lambda_{2}+\lambda_{3}+\lambda_{4}+\lambda_{5}+\lambda_{6}+\lambda_{7} \\
+\lambda_{8}+\lambda_{9}+\lambda_{10}+\lambda_{11}+\lambda_{13}
\end{array}\right) \Delta t\right) P_{14} \mid \\
& +\mu_{1} \Delta t P_{1}(t)+\mu_{2} \Delta t P_{2}(t)+\mu_{3} \Delta t P_{3}(t) \\
& +\mu_{4} \Delta t P_{4}(t)+\mu_{5} \Delta t P_{5}(t)+\mu_{6} \Delta t P_{6}(t) \\
& +\mu_{7} \Delta t P_{7}(t)+\mu_{8} \Delta t P_{8}(t)+\mu_{9} \Delta t P_{9}(t) \\
& +\mu_{10} \Delta t P_{10}(t)+\mu_{11} \Delta t P_{11}(t)+\mu_{12} \Delta t P_{12}(t) \\
& +\mu_{13} \Delta t P_{13}(t)
\end{aligned}
$$

According to transition state diagram (Figure 3), weights of synaptic links are as follows:

$$
\begin{aligned}
& w_{11}=1-\mu_{1} \Delta t, w_{1(14)}=\mu_{1} \Delta t, \\
& w_{12}=w_{13}=w_{14}=w_{15}=w_{16}=w_{17}=0 \\
& w_{18}=w_{19}=w_{1(10)}=w_{1(11)}=w_{1(12)}=w_{1(13)}=0 \\
& w_{21}=0, w_{22}=1-\mu_{2} \Delta t, w_{2(14)}=\mu_{2} \Delta t, \\
& w_{23}=w_{24}=w_{25}=w_{26}=w_{27}=w_{28}=0 \\
& w_{29}=w_{2(10)}=w_{2(11)}=w_{2(12)}=w_{2(13)}=0 \\
& w_{31}=w_{32}=0, w_{3(14)}=\mu_{3} \Delta t, \\
& w_{33}=1-\mu_{3} \Delta t, \\
& w_{34}=w_{35}=w_{36}=w_{37}=w_{38}=0 \\
& w_{39}=w_{3(10)}=w_{3(11)}=w_{3(12)}=w_{3(13)}=0 \\
& w_{41}=w_{42}=w_{43}=0, w_{4(14)}=\mu_{4} \Delta t, \\
& w_{44}=1-\mu_{4} \Delta t, \\
& w_{45}=w_{46}=w_{47}=w_{48}=0 \\
& w_{49}=w_{4(10)}=w_{4(11)}=w_{4(12)}=w_{4(13)}=0 \\
& w_{55}=1-\mu_{5} \Delta t, w_{5(13)}=\mu_{5} \Delta t, \\
& w_{51}=w_{52}=w_{53}=w_{54}=w_{56}=w_{57}=0 \\
& w_{58}=w_{59}=w_{5(10)}=w_{5(11)}=w_{5(12)}=w_{5(13)}=0 \\
& w_{66}=1-\mu_{6} \Delta t, w_{6(13)}=\mu_{6} \Delta t, \\
& w_{61}=w_{62}=w_{63}=w_{64}=w_{65}=w_{67}=0 \\
& w_{68}=w_{69}=w_{6(10)}=w_{6(11)}=w_{6(12)}=w_{6(13)}=0 \\
& w_{77}=1-\mu_{7} \Delta t, w_{7(13)}=\mu_{7} \Delta t, \\
& w_{71}=w_{72}=w_{73}=w_{74}=w_{75}=w_{76}=0 \\
& w_{78}=w_{79}=w_{7(10)}=w_{7(11)}=w_{7(12)}=w_{7(13)}=0 \\
& w_{88}=1-\mu_{8} \Delta t, w_{8(13)}=\mu_{8} \Delta t, \\
&
\end{aligned}
$$

$$
\begin{aligned}
& w_{81}=w_{82}=w_{83}=w_{84}=w_{85}=w_{86}=0 \\
& w_{87}=w_{89}=w_{8(10)}=w_{8(11)}=w_{8(12)}=w_{8(13)}=0 \\
& w_{91}=\lambda_{1} \Delta t, w_{92}=\lambda_{2} \Delta t, w_{93}=\lambda_{3} \Delta t, \\
& w_{94}=\lambda_{4} \Delta t, w_{95}=\lambda_{5} \Delta t, w_{96}=\lambda_{6} \Delta t \text {, } \\
& w_{97}=\lambda_{7} \Delta t, w_{98}=\lambda_{8} \Delta t \text {, } \\
& w_{99}=1-\left(\begin{array}{l}
\lambda_{1}+\lambda_{2}+\lambda_{3}+\lambda_{4}+\lambda_{5}+\lambda_{6}+\lambda_{7} \\
+\lambda_{8}+\lambda_{10}+\lambda_{11}+\lambda_{12}+\mu_{9}
\end{array}\right) \Delta t \\
& w_{9(10)}=\lambda_{10} \Delta t, w_{9(11)}=\lambda_{11} \Delta t \text {, } \\
& w_{9(12)}=\lambda_{12} \Delta t, w_{9(14)}=\mu_{9} \Delta t \text {, } \\
& w_{(10) 1}=w_{(10) 2}=w_{(10) 3}=w_{(10) 4}=w_{(10) 5}=w_{(10) 6}=0 \\
& w_{(10) 7}=w_{(10) 8}=w_{(10) 9}=w_{(10)_{(11)}}=w_{(10)(12)}=w_{(10)(13)}=0 \\
& w_{(10)(10)}=1-\mu_{10} \Delta t, w_{(10)(14)}=\mu_{10} \Delta t \text {, } \\
& w_{(11) 1}=w_{(11) 2}=w_{(11) 3}=w_{(11) 4}=w_{(11) 5}=w_{(11) 6}=0 \\
& w_{(11) 7}=w_{(11) 8}=w_{(11) 9}=w_{(11)(10)}=w_{(11)(12)}=w_{(11)(13)}=0 \\
& w_{(11)(11)}=1-\mu_{11} \Delta t, w_{(11)(14)}=\mu_{11} \Delta t \text {, } \\
& w_{(12) 1}=w_{(12) 2}=w_{(12) 3}=w_{(12) 4}=w_{(12) 5}=w_{(12) 6}=0 \\
& w_{(12) 7}=w_{(12) 8}=w_{(12) 9}=w_{(12)(10)}=w_{(12)(11)}=w_{(12)(13)}=0 \\
& w_{(12)(12)}=1-\mu_{12} \Delta t, w_{(12)(14)}=\mu_{12} \Delta t \text {, } \\
& w_{(13) 1}=\lambda_{1} \Delta t, \quad w_{(13) 2}=\lambda_{2} \Delta t, w_{(13) 3}=\lambda_{3} \Delta t \text {, } \\
& w_{(13) 4}=\lambda_{4} \Delta t, \quad w_{(13) 5}=\lambda_{5} \Delta \mathrm{t} \text {, } \\
& w_{(13) 6}=\lambda_{6} \Delta t, w_{(13) 7}=\lambda_{7} \Delta t, w_{(13) 8}=\lambda_{8} \Delta t \text {, } \\
& w_{(13)(10)}=\lambda_{10} \Delta t, \quad w_{(13)(11)}=\lambda_{11} \Delta t \text {, } \\
& w_{(13)(12)}=\lambda_{12} \Delta t, w_{(13)(14)}=\mu_{13} \Delta t \text {, } \\
& w_{(13)(13)}=1-\left(\begin{array}{l}
\lambda_{1}+\lambda_{2}+\lambda_{3}+\lambda_{4}+\lambda_{5}+\lambda_{6}+\lambda_{7} \\
+\lambda_{8}+\lambda_{10}+\lambda_{11}+\lambda_{12}+\mu_{13}
\end{array}\right) \Delta t \\
& w_{(14) 1}=\lambda_{1} \Delta t, \quad w_{(14) 2}=\lambda_{2} \Delta t, \quad w_{(14) 3}=\lambda_{3} \Delta t \text {, } \\
& w_{(14) 4}=\lambda_{4} \Delta t, \quad w_{(14) 5}=\lambda_{5} \Delta t, \quad w_{(14) 6}=\lambda_{6} \Delta t \text {, } \\
& w_{(14) 7}=\lambda_{7} \Delta t, w_{(14) 8}=\lambda_{8} \Delta t, w_{(14) 9}=\lambda_{9} \Delta t \text {, } \\
& w_{(14)(10)}=\lambda_{10} \Delta t, \quad w_{(14)(11)}=\lambda_{11} \Delta t \text {, } \\
& w_{(14)(13)}=\lambda_{13} \Delta t \\
& w_{(14)(14)}=1-\left(\begin{array}{l}
\lambda_{1}+\lambda_{2}+\lambda_{3}+\lambda_{4}+\lambda_{5}+\lambda_{6}+\lambda_{7} \\
+\lambda_{8}+\lambda_{9}+\lambda_{10}+\lambda_{11}+\lambda_{13}
\end{array}\right) \Delta t
\end{aligned}
$$

Published By: Blue Eyes Intelligence Engineering and Sciences Publication (c) Copyright: All rights reserved. 


\section{BACK PROPAGATION NEURAL NETWORK ALGORITHM OF THE SYSTEM}

Step 1: Assume total number of inputs and outputs

Step 2: Set desired output

Step 3: Input the number of neurons in hidden layer.

Step 4: Initialize the synaptic link's weight matrices

[W]: Weights of synaptic links connecting input and hidden layer.

[V]: Weights of synaptic links connecting hidden and output layer.

Step 5: Evaluate inputs of hidden layer using

$$
\mathrm{I}_{\mathrm{h}}=\mathrm{W}^{\mathrm{T}} * \mathrm{O}_{\mathrm{i}}
$$

$\left[\mathrm{W}^{\mathrm{T}}\right]$ : Transpose of weight matrix connecting input and hidden layer.

$\left[\mathrm{O}_{\mathrm{i}}\right]$ : Output of Input Layer

Step 6: Evaluate output of hidden layer using Sigmoidal function, i.e. $O_{h}=\left\{\frac{1}{1+e^{-I_{h_{i}}}}\right\}$

Step 7: Evaluate inputs of output layer using $\mathrm{I}_{\mathrm{o}}=\mathrm{V}^{\mathrm{T}} * \mathrm{O}_{\mathrm{h}}$

$\left[\mathrm{V}^{\mathrm{T}}\right]$ : Transpose of weight matrix connecting hidden and output layer.

$\left[\mathrm{O}_{\mathrm{h}}\right]$ : Output of hidden Layer

Step 8: Evaluate output of output layer using Sigmoidal function, i.e. $O_{o}=\left\{\frac{1}{1+e^{-I_{0 i}}}\right\}$

Step 9: Compare evaluated output (in step 8) with

Target output (in step 2), then evaluate the error

Step 10: If error < tolerance

Then End the learning process.

Else

Update weight matrices [V] and [W] using error correction gradient descent method

Increment the epochs by 1

go to Step 5

else End the learning process

Inputs, $X_{i}$, demonstrate in Neural network structure (Figure

$1)$, express by following equations:

$$
X_{i}=P_{i}(t) \text {; where } i=1,2, \ldots, 14
$$

The outputs of neurons, $Y_{i}$, of considered system represent by following equations:

$$
Y_{i}=P_{i}(t+\Delta t) \text {, where } i=1,2, \ldots, 14
$$

Using transition states diagram (Figure 3), authors established following output equations:

$$
\begin{aligned}
& Y_{1}=w_{11} X_{1}+w_{91} X_{9}+w_{(13) 1} X_{(13)}+w_{(14) 1} X_{14} \\
& Y_{2}=w_{22} X_{2}+w_{92} X_{9}+w_{(13) 2} X_{(13)}+w_{(14) 2} X_{14} \\
& Y_{3}=w_{33} X_{3}+w_{93} X_{9}+w_{(13) 3} X_{(13)}+w_{(14) 3} X_{14}
\end{aligned}
$$

$$
\begin{aligned}
Y_{4}= & w_{44} X_{4}+w_{94} X_{9}+w_{(13) 4} X_{(13)}+w_{(14) 4} X_{14} \\
Y_{5}= & w_{55} X_{5}+w_{95} X_{9}+w_{(13) 5} X_{(13)}+w_{(14) 5} X_{14} \\
Y_{6}= & w_{66} X_{1}+w_{96} X_{9}+w_{(13) 6} X_{(13)}+w_{(14) 6} X_{14} \\
Y_{7}= & w_{77} X_{7}+w_{97} X_{9}+w_{(13) 7} X_{(13)}+w_{(14) 7} X_{14} \\
Y_{8}= & w_{88} X_{8}+w_{98} X_{9}+w_{(13) 8} X_{13}+w_{(14) 8} X_{14} \\
Y_{9}= & w_{99} X_{9}+w_{(14) 9} X_{14} \\
Y_{(10)}= & w_{(10)(10)} X_{(10)}+w_{9(10)} X_{9}+w_{(13)(10)} X_{(13)} \\
& +w_{(14)(10)} X_{14} \\
& +w_{8(14)} X_{8}+w_{9(14)} X_{9}+w_{(10)(14)} X_{10}+w_{(11)(14)} X_{11} \\
Y_{(11)}= & w_{(11)(11)} X_{(11)}+w_{9(11)} X_{9}+w_{(13)(11)} X_{(13)} \\
& +w_{(14)(11)} X_{(14)}+w_{(13)(14)} X_{13} \\
Y_{14}= & w_{(14)(14)} X_{14}+w_{1(14)} X_{1}+w_{2(14)} X_{2}+w_{3(14)} X_{3} \\
+ & w_{4(14)} X_{4}+w_{5(14)} X_{5}+w_{6(14)} X_{6}+w_{7(14)} X_{7} \\
Y_{(12)}= & w_{(12)(12)} X_{(12)}+w_{9(12)} X_{9}+w_{(13)(12)} X_{(13)} \\
Y_{13}= & w_{(10)(13)} X_{13}+w_{(14)(13)} X_{14} \\
+ &
\end{aligned}
$$

Transition states, whether the system is in working mode or in failure mode, can be classified as up and down states respectively. The up and down state probabilities of the system state combine distinctly from (17)-(30) and represent as follows:

$$
\begin{aligned}
& P_{\text {up }}(t)=Y_{9}+Y_{13}+Y_{14} \\
& P_{\text {down }}(t)=1-P_{\text {up }}
\end{aligned}
$$

The reliability and profit function of the considered system can be expressed as:

Reliabilit y $=Y_{9}+Y_{13}+Y_{14}$

$G(t)=C_{1} \times P_{u p}-C_{2} \times t$

where $C_{1}$ : revenue cost

$\mathrm{C}_{2}$ : repair cost per unit time

$\mathrm{P}_{\mathrm{up}}(\mathrm{t})$ : Probability of operable states

Published By:

Blue Eyes Intelligence Engineering

and Sciences Publication

(C) Copyright: All rights reserved.

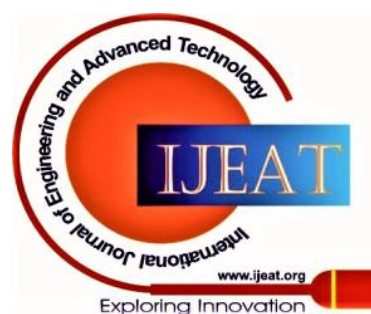




\section{NUMERICAL EXAMPLE}

Following data for repairable system are assumed by authors, to analyze the results

$$
\mu_{i}=1 ; \lambda_{i}=0.01 ; a=0.8
$$

$>$ Equation (32) and (33) yields unreliability and reliability of the system by BP neural network technique. Table I and Figure 4 exhibit the desired reliability, i.e. approximate 1 , up to $10^{-4}$ error tolerance in tabular and graphical form respectively. Figure 5 shows the corresponding unreliability.

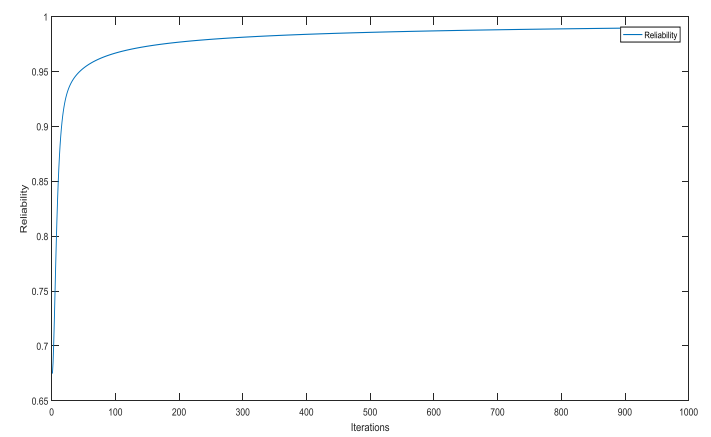

Fig. 4: Optimize Reliability with Iteration

Table I. Reliability with iteration

\begin{tabular}{|c|c|c|c|}
\hline $\begin{array}{c}\text { Iterat } \\
\text { ions }\end{array}$ & Pup & Pdown & $\begin{array}{c}\text { Total } \\
\text { =Pup+Pdown }\end{array}$ \\
\hline 1 & 0.6748 & 0.5001 & 1.1749 \\
\hline 25 & 0.9325 & 0.0218 & 0.9543 \\
\hline 50 & 0.9532 & 0.0191 & 0.9723 \\
\hline 100 & 0.9669 & 0.0165 & 0.9834 \\
\hline 150 & 0.9731 & 0.0146 & 0.9877 \\
\hline 200 & 0.9769 & 0.0133 & 0.9902 \\
\hline 250 & 0.9794 & 0.0123 & 0.9917 \\
\hline 300 & 0.9813 & 0.0116 & 0.9929 \\
\hline 350 & 0.9828 & 0.0108 & 0.9936 \\
\hline 400 & 0.984 & 0.0102 & 0.9942 \\
\hline 450 & 0.9849 & 0.0097 & 0.9946 \\
\hline 500 & 0.9858 & 0.0092 & 0.995 \\
\hline 550 & 0.9865 & 0.0089 & 0.9954 \\
\hline 600 & 0.9871 & 0.0085 & 0.9956 \\
\hline 650 & 0.9876 & 0.0082 & 0.9958 \\
\hline 700 & 0.9881 & 0.008 & 0.9961 \\
\hline 750 & 0.9885 & 0.0077 & 0.9962 \\
\hline 800 & 0.9889 & 0.0075 & 0.9964 \\
\hline 850 & 0.9893 & 0.0073 & 0.9966 \\
\hline 900 & 0.9896 & 0.0071 & 0.9967 \\
\hline 950 & 0.9899 & 0.0069 & 0.9968 \\
\hline 968 & 0.99 & 0.0068 & 0.9968 \\
\hline
\end{tabular}

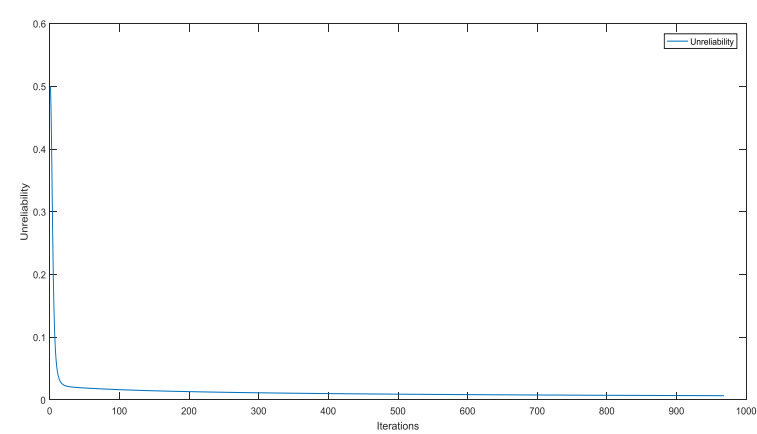

Fig. 5: Minimize Unreliability with Iteration

One can obtain the various values of the profit function of the system using equation (34). Consider the revenue cost $C_{1}=100$ units and different values of repair cost, $C_{2}=30,50,80$ units for comparison and analyzing the profit function. Profit function values are evaluated that are listed in Table II and represented graphically in Figure 6.

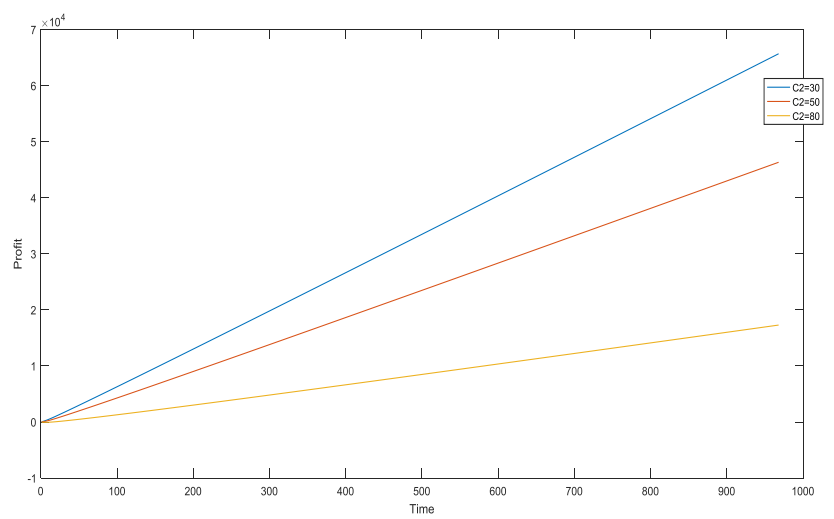

Fig 6 : Time vs Comparative profit

Table II. Time Vs Comparative profit

\begin{tabular}{|c|c|c|c|}
\hline \multirow{2}{*}{$\begin{array}{l}\text { Time/ } \\
\text { epochs }\end{array}$} & \multicolumn{3}{|c|}{ Profit Function Value (units) } \\
\hline & $\mathrm{C} 2=80$ & $\mathrm{C} 2=50$ & $\mathrm{C} 2=30$ \\
\hline 1 & -12.5185 & 17.4815 & 37.4815 \\
\hline 50 & 483.8873 & 1983.8873 & 2983.8873 \\
\hline 100 & 1290.4979 & 4290.4979 & 6290.4979 \\
\hline 150 & 2142.3422 & 6642.3422 & 9642.3422 \\
\hline 200 & 3018.1094 & 9018.1094 & 13018.1094 \\
\hline 250 & 3909.2754 & 11409.2754 & 16409.2754 \\
\hline 300 & 4811.4027 & 13811.4027 & 19811.4027 \\
\hline 350 & 5721.8351 & 16221.8351 & 23221.8351 \\
\hline 400 & 6638.8349 & 18638.8349 & 26638.8349 \\
\hline 450 & 7561.1926 & 21061.1926 & 30061.1926 \\
\hline 500 & 8488.0270 & 23488.0270 & 33488.0270 \\
\hline 550 & 9418.6726 & 25918.6726 & 36918.6726 \\
\hline 600 & 10352.6128 & 28352.6128 & 40352.6128 \\
\hline 650 & 11289.4370 & 30789.4370 & 43789.4370 \\
\hline 700 & 12228.8127 & 33228.8127 & 47228.8127 \\
\hline 750 & 13170.4659 & 35670.4659 & 50670.4659 \\
\hline 800 & 14114.1682 & 38114.1682 & 54114.1682 \\
\hline 850 & 15059.7266 & 4055 & 20 \\
\hline
\end{tabular}

Published By: Blue Eyes Intelligence Engineering and Sciences Publication 


\begin{tabular}{|l|l|l|l|}
900 & 16006.9762 & 43006.9762 & 61006.9762 \\
\hline 950 & 16955.7749 & 45455.7749 & 64455.7749 \\
\hline 968 & 17297.6974 & 46337.6974 & 65697.6974 \\
\hline
\end{tabular}

\section{CONCLUSION}

In current scenario, neural network's applications associate with diverse fields such as image processing, optimization of system working, machine learning etc.

It consists of many techniques; one of them is back propagation neural networks to optimize the output of the system. EVM is an electronic machine that takes care of casting and counting of votes during election process of any democratic country. It incorporates many discrete components with reliability factors. Determining the accuracy of the system reliability is a key factor that maximizes system availability.

As shown in Table I, the reliability of the system is initially at 0.6748. After increment in iterations/ epochs, the weights of synapses used as failures/repairs are modified, using gradient descent method of back propagation of neural networks. The reliability of system attains up to 0.99 in 968 iterations with $10^{-4}$ tolerance. The iteratively improvement in reliability of the system reveals the importance of the BPNN technique especially in the complex engineering system.

On examination the results of Equation (34) of profit function that represents in Table II and graphically in Figure 6. It steadily increases with respect to iterations. One can also depict the comparison of profit with different values of repair cost. One can easily read the table II, when repair cost is less i.e. 30 units, profit function has maximum value each time/ epochs which can stimulate operational managers to make the decision.

Therefore, the neural networks technique for optimizing the output can be an adequate and efficient way to compute as well as improve the reliability parameters of the system using the learning and updating weight mechanism.

The analysis done in this paper ensures that neural network approach helps in attaining system reliability with high precision. Hence it enhances reliability and reinforce the system utility for real time applications.

\section{REFERENCES}

1. A. P. Gangwar, "Issues and Challenges in Electronic Voting and Direct Recording Electronic Voting Systems", International Journal of Advanced Research in Computer Science and Software Engineering, 439-443, 2015.

2. Ashok Kumar D., U. S., "A Novel design of Electronic Voting System Using Fingerprint", International Journal of Innovative Technology \& Creative Engineering (ISSN:2045-8711), 12-19, 2011.

3. D.R. Cox, "Analysis of non-Markovian Stochastic processes by the inclusion of Supplementary variables" Proc.Comb.Phill. Soc. 51, 1955, pp 433-441.

4. Dip Jyoti Bhuyan, "Effectiveness of Electronic Voting Machine in the Electoral System of India: New Opportunities and Challenges" International Journal of Recent Technology and Engineering (IJRTE) ISSN: 2277-3878, Volume-8 Issue-2, July 2019

5. Hinton, G. E.; Osindero, S.; Teh, Y. "A fast learning algorithm for deep belief nets", Neural Computation. 18 (7):527-1554. CiteSeerX 10.1.1.76.1541. doi:10.11 62/neco.2006.18.7.1527. PMID 167645132006

6. Igor Bazovsky, Reliability Theory and Practice, PHI Englewood cliff, NJ., 1961.

7. J. Singh and P. Goyal, "Availability analysis of a standby complex system having imperfect switch over device" Microelectron Reliab. 35, 1996, 285-288

8. M. Ram, S.B. Singh and V.V. Singh, "Stochastic analysis of a standby system with waiting repair strategy", IEEE transactions on systems, man, and cybernetics: systems, 43(3), 2013.

9. G. Levitin, L. Xing and Y. Dai, "Reliability versus expected mission cost and uncompleted work in heterogeneous warm standby multiphase systems", IEEE Transactions on Systems, Man, and Cybernetics: Systems, 47(3), 2017, pp 462 - 473.

10. S. Rajasekaran and G.A. Vijaylakshmi Pai, Neural Networks, Fuzzy Logic, and Genetic Algorithm, PHI, 2004.

11. N. Karunanithi, D. Whitley and Y.K. Malaiya, "Using neural networks in reliability", IEEE, 9, 1992, pp. 53-59.

12. I. Lakshman and S. Ramaswamy "An Artificial Neural-Network Approach to Software Reliability Growth Modeling", Procedia Computer Science. 57, 2015, pp. 695-702.

13. V.Rao and Goswam, "E- Voting: An analysis of Security Issues in EVM" International Journal on Emerging Technologies, 403-405, 2017.

14. V. Srivastava and Tere,G "An analysis of Electronic Voting Machine for its Effectiveness" International Journal of Computing Experiments, 8-14, 2016

15. Warren S. McCulloch and Walter H.Pitts, "A logical Calculus of Ideas Immanent in nervous Activity", Bulletin of Mathematical Biophysics, vol. 5, pp 115-133, 1943.

16. Xiang-Yu Li, Hong-Zhong Huang, Yan-Feng Li, "Reliability analysis of phased mission system with non-exponential and partially repairable components", Reliability Engineering and System Safety 175, pp. 119-127, 2018.

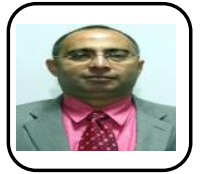

\section{AUTHORS PROFILE}

Dr. C. M. Batra is a Professor and Head of the Department of Applied Sciences, KIET Group of Institutions, Ghaziabad. He has twenty five years experience of teaching and research. He has received Ph.D. in Mathematics and Diploma in Computer Sciences. He is a Life member of ISTE. He has been published several research papers in various national and international Journals. He has co-authored book related to Engineering topics. He has organized and attended many International Conferences, Short term courses, faculty development programs, workshops to enhance the research work and knowledge. His research interest includes Soft Computing and Reliability Engineering.

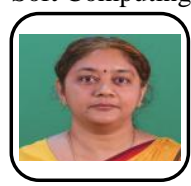

Dr. Ritu Gupta is a Professor (Mathematics) in the Department of Applied Sciences, KIET Group of Institutions, Ghaziabad. She has acquired M.Phil. and Ph.D degrees in Mathematics. She has twenty one years of teaching experience as well as actively participates in institute academic and administrative activities. She is life member of Indian Society for Technical Education (ISTE). She has published many research papers in National and International journals and also co-author of books with International and National publishers. She received accolades for excellence in teaching and is contributing actively to organize seminars, conferences and symposiums in institute. Her research interests include Reliability theory and Soft Computing.

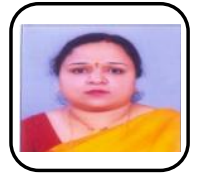

Dr. Ekata is a Professor in the Department of Applied Sciences, KIET Group of Institutions, Ghaziabad. She has around twenty years of teaching and research experience. She has received versatile degrees, Ph.D. in Mathematics and Master in Computer Application. She is a senior member of IEEE-Computational Intelligence Society and life member of ISTE. She is a regular reviewer of International Journals. She is an active member of organizing committee of many seminars and conferences. She has co-authored two books on different courses of Applied Mathematics. She has published several research papers in reputed International and National Journals. Her area of research is Soft Computing and Reliability Theory.

Published By:

Blue Eyes Intelligence Engineering and Sciences Publication (c) Copyright: All rights reserved.

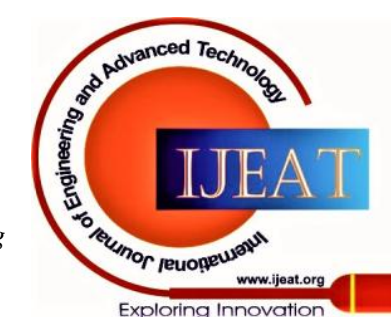

